\title{
An experimental approach to exploring the feasibility of unmanned aerial vehicle and thermal imaging in terrestrial and arboreal mammals research
}

\author{
Dede Aulia Rahman ${ }^{1,2^{*}}$, Yudi Setiawan ${ }^{1,2}$, Arif $K$ Wijayanto $^{1}$, Ahmad Abdul Aziz Fathur \\ Rahman $^{2}$, and Trisna Rizky Martiyani ${ }^{2}$ \\ ${ }^{1}$ Department of Forest Resources Conservation and Ecotourism, Faculty of Forestry and \\ Environment, IPB University, Bogor, 16680 Indonesia. \\ ${ }^{2}$ Tropical Biodiversity Conservation Program, Faculty of Forestry and Environment, IPB University, \\ Bogor, 16680, Indonesia.
}

\begin{abstract}
The visual camouflage of many species living in the dense cover of the tropical rainforest become obstacles to conducting species monitoring. Unmanned aerial vehicles (drones) combined with thermal infrared imaging (TIR) can rapidly scan large areas from above and detect wildlife that has a body temperature that contrasts with its surrounding environment. This research tested the feasibility of DJI Mavic 2 Enterprise Dual with FLIR as aerial survey platforms to detect terrestrial and arboreal mammals in the five tree density classes in the remaining natural environment on the IPB University campus. This study demonstrated that large-size terrestrial mammal thermal signatures are visible in sparse vegetation at daytime and in the area under the canopy at night monitoring. In contrast, arboreal mammals were better detected in at early morning and night. Survey timing highly influenced the results - the best quality thermal images were obtained at sunrise, late evening, and at night. The drones allow safe operation at low altitudes with low levels of disturbance to animals. Both terrestrial and arboreal mammals are well detected and easily identified when the drone is flying at an altitude < 50 m HAGL. Our preliminary results indicated that thermal surveys from drones are a promising method.
\end{abstract}

\section{Introduction}

Wildlife monitoring is an essential aspect of biodiversity conservation efforts $[1,2]$. A major challenge in studying mammals in the field is finding them because they are often difficult to detect visually or have nocturnal behaviors. On the other hand, ground-based observation of wildlife is often limited by access and topography. Aerial surveys are often the only practical way to detect and estimate a target species' numbers. In the past decade, unmanned aerial vehicle technology has entered a new era in wildlife research and

\footnotetext{
* Corresponding author: dede.auliarahman@gmail.com
} 
monitoring [3-5]. Imagery from aerial surveys has been useful for surveys of animals in open areas; for example, counting elephant [6], Arctic birds [7], and hippos [8]. Aerial surveys to monitor wildlife in tropical areas are still rare because species live in rainforest habitat, where a thick canopy shades them from visual detection. Small airplanes have been used in a minimal number for aerial detection and population surveys of a range of wildlife species in these habitats, for example, orangutan [9], chimpanzee [10], howler monkey [11], and spider monkey [12].

This research uses consumer unmanned aerial vehicles (UAVs, commonly referred to as "drones") that fitted with a thermal infrared (TIR) camera to monitor mammals in the wild. This way is an opportunity that has only emerged in the last decade and could provide a solution for monitoring animals that are under heavy canopy. Recently, there have been technical improvements, particularly in terms of spatial and thermal resolution, since TIR cameras' initial use for surveying and monitoring large mammals in the late 1960s [13]. A substantial reduction in equipment cost has accompanied this application, enabling its use on a broader scale. The increase in thermal resolution from the time of its initial use to the present has made it possible to make TIR imaging one of the most suitable methods for animal monitoring activities. Lower costs, safe operation at night, and higher animal detection are the advantages of aerial survey activities that combine UAV technology with TIR.

However, few studies have assessed UAVs' combined potential and multispectral imagery (standard RGB and thermal infrared) to monitor tropical rainforest mammals. For the first time in this study, this research assessed these technologies' potential for surveying mammals who spend the majority of their lives on grounds (terrestrial) and trees (arboreal) in tropical rainforest Indonesia. Its main interest is evaluating the UAVs-TIR sensor system's performance in detecting mammals under various environmental conditions and determining several flight parameters and the response of animals to the flight of this technology.

\section{Method}

\subsection{Study area}

The study was located in the remaining of the natural forest on the IPB University campus, Indonesia ( $6^{\circ} 30^{\prime}-6^{\circ} 45^{\prime} \mathrm{S}$ and $106^{\circ} 30^{\prime}-106^{\circ} 45^{\prime} \mathrm{E}$; Fig. 1$)$, a home and essential habitat of various species surrounded by urban areas and human activity. The area of the campus is $267 \mathrm{ha}$, and the terrain is flat. Less than $40 \%$ of the area is covered by various types of trees of different age and canopy cover classes. The most common mammals in the remaining forest are long-tailed macaque (Macaca fascicularis), pangolin (Psiittacula alexandri), Malayan porcupine (Hystrix brachyura), Asian palm civets (Paradoxurus hermaphroditus), Javan mongoose (Herpestes javanica), as well as several species of rats, squirrels, and bats. 


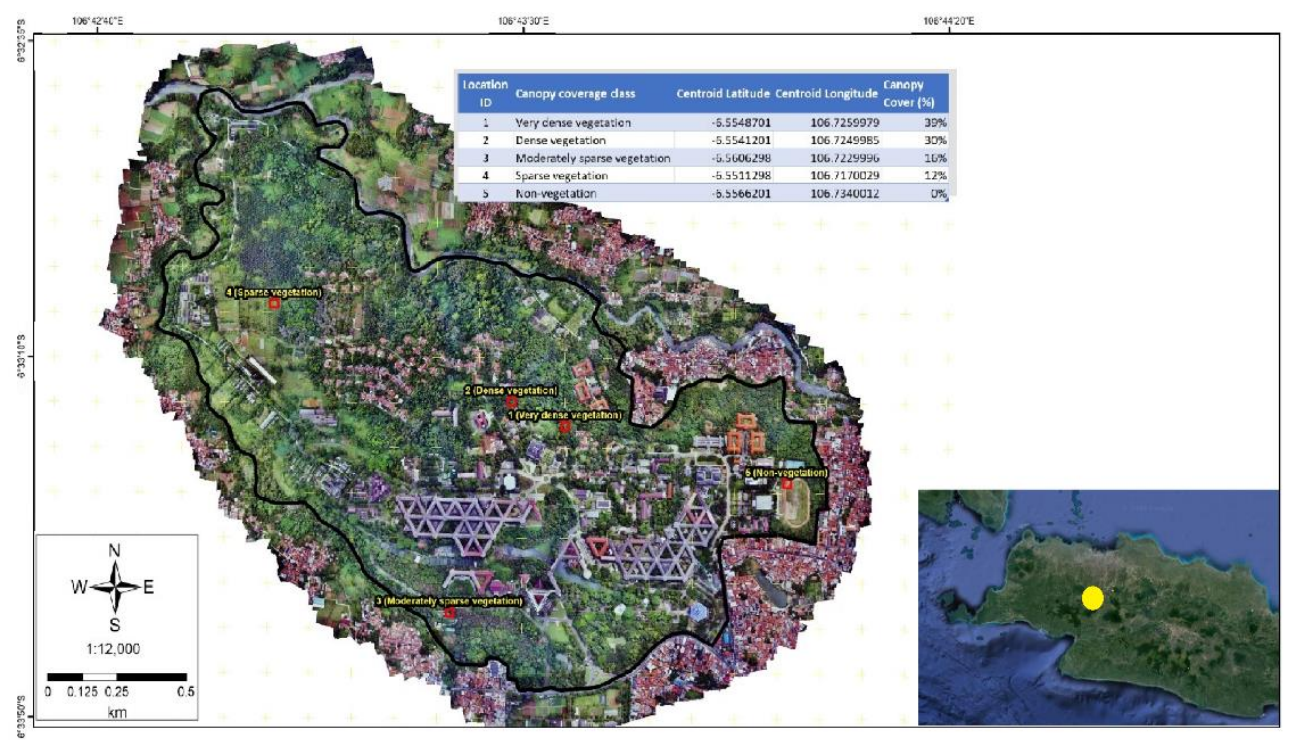

Fig 1. Location of square plots (red line polygons) of this study area (Rahman et al. 2020).

\subsection{Data collection}

\subsubsection{Drone platform}

This research used DJI Mavic 2 Enterprise Dual with FLIR as aerial survey platforms (hereafter "drones"). The Mavic 2 Enterprise Dual is equipped with a standard camera and infrared sensor. Measurement of temperature in the center of the field of view is possible on an infrared camera. Images or videos recorded on the camera can be viewed independently (just 1080P RGB or just thermal) or directly. FLIR MSX (multispectral dynamic imaging) on the drone with an easily visible light spectrum, were able to show the heat difference between objects recorded. The two spectra that present two different views of the object recorded on each camera sensor can be seen in one view by the operator. Equipped with OcuSync 2.0, this technology can easily transmit a 1080P video even through a concrete barrier with a transmission range of up to 5 miles. The time, date, and GPS location of every image or video recorded in flight are recorded on GPS timestamping. This additional data is critical information that shows the time and location of the object recorded, either in the form of images or videos. Flights can be carried out for up to 31 minutes with minimal sound and move quickly by taking advantage of DJI's efficient propulsion system. The Mavic 2 Enterprise can move at a maximum speed of $44 \mathrm{mph}$ to keep pace with target objects while moving. In cold weather conditions, the battery of Mavic 2 Enterprise Dual can warm up on its own, increasing its usability in various weather conditions. In addition to these advantages, the Mavic 2 Enterprise also has a new "Discrete Mode", making it possible to turn off all the aircraft's LEDs so the user can fly it for missions that require undetectable stealth mode flight. This research used an Apple 7.9" iPad (128GB, Wi-Fi + 4G LTE) as our live-feed screen for both systems.

\subsubsection{Flight plans and parameters}


The test flights were conducted over five square plot sample areas, ranging in area from 90 x $90 \mathrm{~m}$, and the selected areas are based on the density of canopy cover (Table 1). Flights were carried out from June to August 2020 (24 hours in each day). Ten flights were flown throughout the day, with two repetitions for each plot location to test animal reaction to the UAV passage. The flight paths over five sample areas had parallel transects positioned along the side of the square plots. To provide complete area coverage, this study designed observation paths close to each other. The UAV's technical characteristics, the relatively small field of view of the camera, and the wind conditions at the time of the survey are used as the basis for determining the parallel transect pattern in survey activities. The flight is carried out autonomously after the drone is launched. The flight path is programmed with HORIZONmp software. Through this software, the operator can monitor the drone's flight on the screen at the ground control station (GCS), including battery status information, global positioning system (GPS) coordinates, flight current speed, and altitude drone.

Table 1. Area proportion for each canopy cover class.

\begin{tabular}{|c|c|c|}
\hline Canopy cover class & Area (ha) & Percentage (\%) \\
\hline Non-vegetation & 17.03 & 6.90 \\
\hline Sparse vegetation & 54.61 & 22.11 \\
\hline Moderately sparse vegetation & 43.79 & 17.73 \\
\hline
\end{tabular}

The total flight time varies per hour, between 20-25 minutes. UAVs are flown at an altitude of 10-100 m HAGL (height above ground level) with an average speed of 8.5-11 m $\mathrm{s}^{-1}\left(30.6-39.6 \mathrm{~km} \mathrm{~h}^{-1}\right)$. Central square plots were filmed having each side length of $30 \mathrm{~m}$. To improve detection and estimate the size of each area surveyed, this study creates a buffer width of 90 on each side along the actual flight path.

\subsection{Data analysis}

\subsubsection{Animal visibility}

All images or videos generated from the 10-100 m HAGL flight on a five-square plot sample are collected and then manually checked under the same strict protocol by two trained independent observers. The protocols applied briefly consist of checking the grid overlay on each image or video, observing the image or video from left to right, and from top to bottom, enlarging the image if necessary to locate terrestrial and arboreal mammals and marking them. To analyze images, this research uses GIMP 2.10.6 software (https://www.gimp.org/).

\subsubsection{Behavioral reaction}

Tests for terrestrial and arboreal mammals' reactions were carried out through a series of trials by flying the UAV 10 times along a parallel line $25 \mathrm{~m}$ apart from each other in the morning over their habitat altitude of $<50 \mathrm{~m}$. At the location where the animal was found, a ground observer near the location recorded each visible animal and their reactions when the UAV passes over them. Next, this research analyzed the change in each category of behavior considered as a percentage. The percentage value is defined as the number of animals displaying each behavior category before and after the UAV mission divided by the total number of animals in each group. 


\section{Results and discussion}

\subsection{Animal visibility}

The ten flights demonstrated that small-sized mammals are quite challenging to detect. In contrast, the Javan deer (terrestrial mammal) and long-tailed macaque (arboreal mammal) that have a larger body size are easily visible at an altitude of $<50 \mathrm{~m}$. The two species are found in sparse vegetation and very dense vegetation, which is quite difficult to detect even with a thermal sensor. A possible reason for the non-detection of small terrestrial and arboreal mammals in open habitats compared to dense vegetation by thermal imaging is the combined effect of surface reflection from sunlight and thermal radiation from the canopy and ground. Compared to studies by [14], our study shows the same findings, washed-out thermal images in open areas due to high surface reflectance, making it challenging to distinguish animals from their background. The canopy of trees in the surrounding is a significant barrier for thermal sensors to detect animals.

During daytime monitoring, a thermal camera provides a better depiction of animal morphology, making it easier to identify. However, the images obtained from this monitoring are not reliable when the animals enter the area under the tree canopy environmental conditions with high temperatures disguise animals' presence (Fig. 2). This case applies vice versa with night monitoring. When the ambient temperature drops drastically, the animals become more easily detected even under the tree canopy (Fig. 3). $[15,16]$ found that the early colder hours of the day provided the finest images. But for the record, flying at night increases the risk of accidents on unmanned aerial vehicles and increases when UAVs are flown at low altitudes with risks including hitting trees, concrete walls, extra-high voltage air channel towers, or power lines.
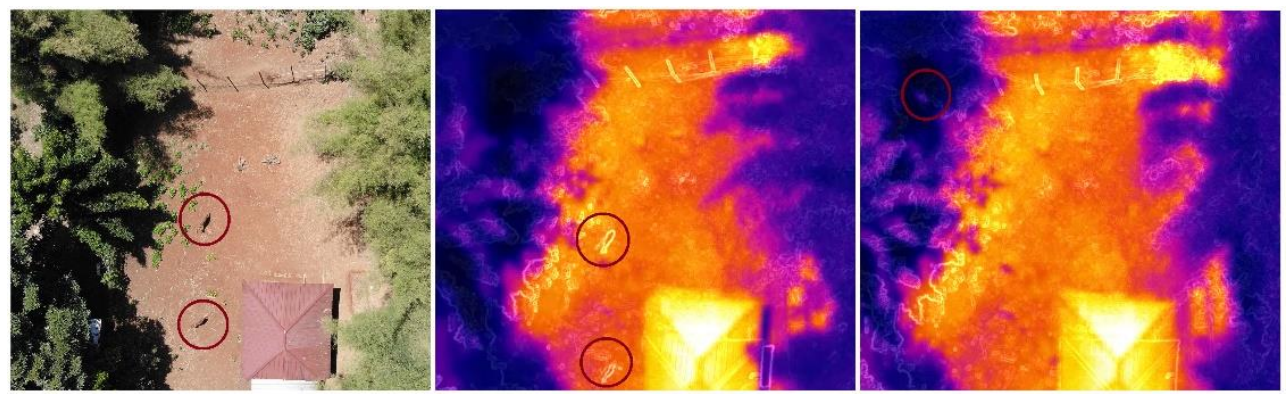

Fig. 2. Aerial images of a Javan deer with visible and thermal imagery (Rahman et al. 2020).

\subsection{Animal reaction to the passage of the UAV}

Group sizes in scanning behavior flights were 3 and 15 animals. On average, before UAV missions, the most common behavioral category shown by Javan deer was foraging (74.46\%), followed by locomotion (18.26\%) and vigilance (5.28\%). During UAV flights, most individuals changed foraging behavior to locomotion and vigilance $(28.80 \%$ and $8.90 \%$, respectively; Fig. 4A). While, for long-tailed macaque, before UAV missions, the most common behavioral category was foraging (43.17\%), followed by locomotion (36.37\%) and vigilance (14.06\%). During UAV flights, most individuals changed foraging behavior to locomotion and vigilance (42.60\% and $28.14 \%$, respectively; Fig. 4B).

Several studies anecdotally monitor UAVs' use and the response shown by species, for example, the study by [17] and [18] who tried to understand the relationship between noise from UAVs, the movement patterns, and stress of target species. In our research, lowering 
the UAV's flying height means that it allows for increased visual detection of the animal, which triggers animal reactions. Thus, research guesses that animals' behavioral responses triggered by auditory rather than visual signals are similar to other studies in primates [19] and large-bodied herbivores [20], revealing the response of the disruption caused by the noise. This guess is shown by the Javan deer's reaction and long-tailed macaque with a more visible behavior change when the noise level of the UAV motor increases with decreasing flying height and increasing the flying speed of UAV. Regardless of the combined influence of the UAV's altitude and speed, these two factors cause the same reaction even when individuals are in the group. During the UAV scanning to see animal reactions to UAV flights, even with a small sample, there was substantial evidence that the long-tailed macaque showed an apparent change in behavior from foraging to being alert and moving. This invention concludes that this behavior is associated with alertness cooperative strategy commonly found in species that live in social groups [21]. Vigilance behavior or movement within the group as a form of reaction to the threat may increase UAV detection probability [22]. The behavioral response of Javan deer to UAV appears to be less reactive. Although these deer live in reasonably large enclosures, this research guesses that the high interaction with humans makes them less reactive to the presence of disturbances. This action may occur in wild populations that have been exposed to human presence for a long time, for example, by human tourism activities around their habitat [23]. However, our study shows that the behavioral changes that occur are not that significant, compared to studies conducted on guanacos [24]. This research suspects that the size of the type of UAV used provides a different level of interference. This study used a small UAV with a much lower noise level so that during the flight, it did not cause significant disruption in the long term for animals. The use of conservation drones in the future must be focused not only to optimize the ability of drones to detect wildlife but also to reduce the level of interference in the target species' activity. 


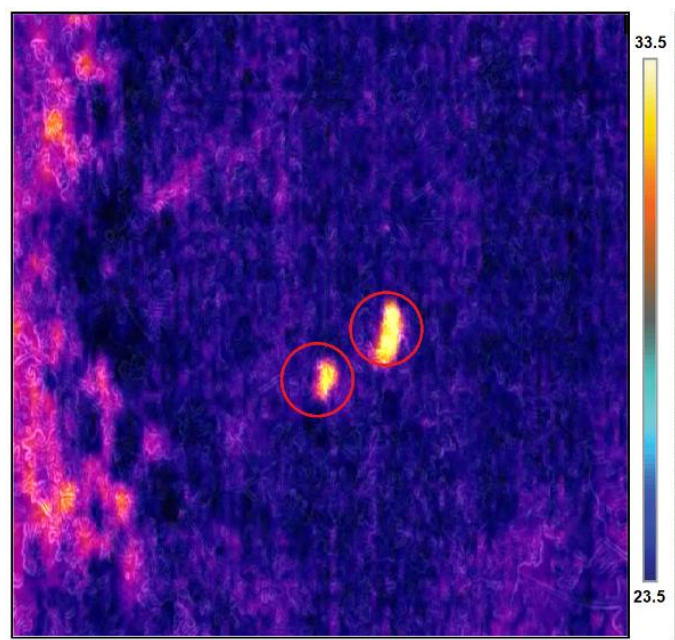

(A)

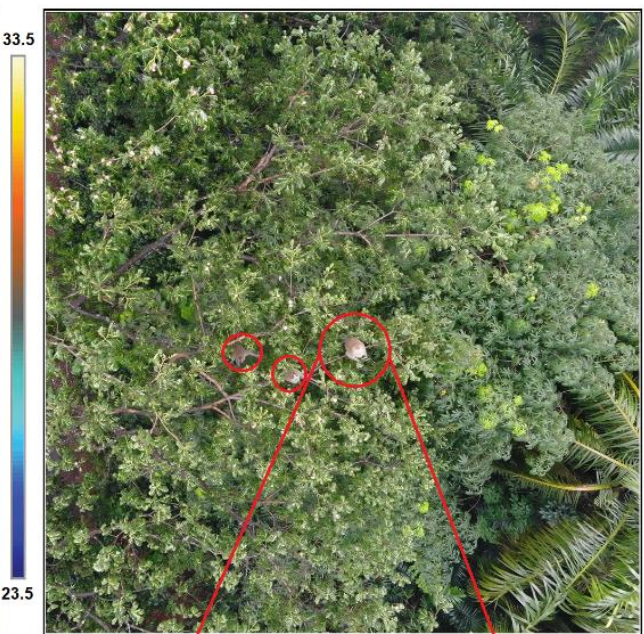

(B)

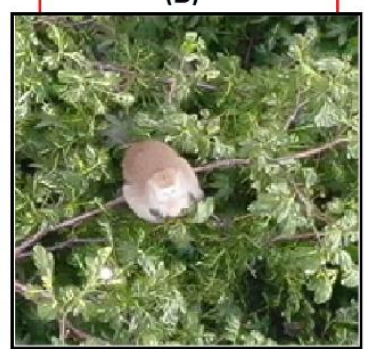

Fig. 3. Video frames demonstrating animals obscured by tree canopy during monitoring at (A) night and (B) daytime (Rahman et al. 2020).

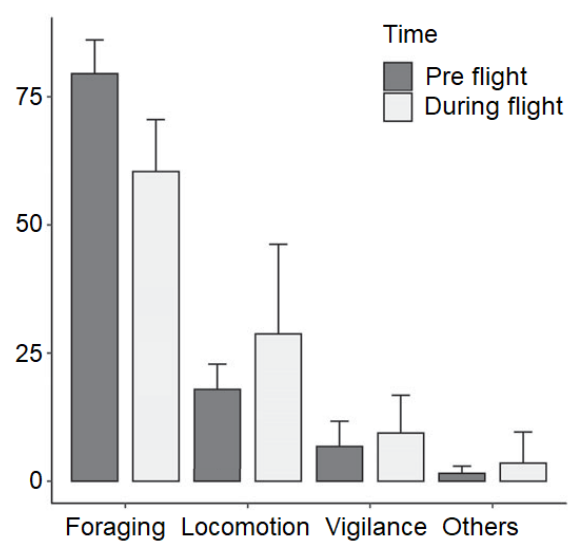

(A)

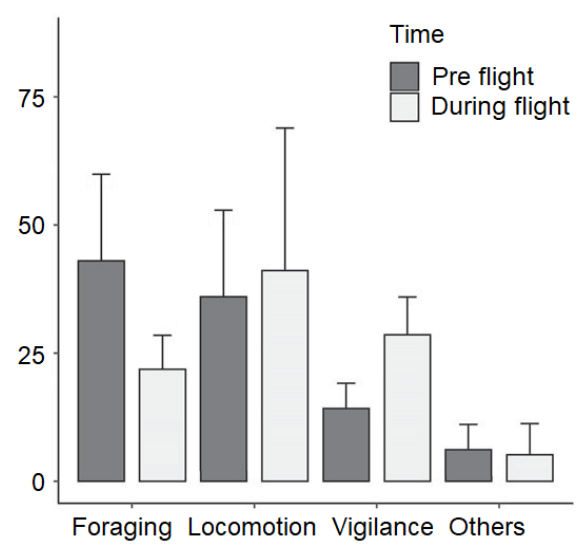

(B)

Fig. 4. The behavioral reaction of (A) Javan deer and (B) long-tailed macaque (average and $\mathrm{SD}$ of the percentages of behavioral categories recorded before and during the scanning behavior flights) (Rahman et al. 2020). 


\section{Conclusion}

Our study represents the first test of unmanned aerial vehicles with thermal infrared imagery to survey tropical rainforest terrestrial and arboreal mammals in Indonesia. This tool can be used in monitoring activities of large terrestrial and arboreal animals, whether living in open areas or habitats with high-density forest stands. UAV with TIR offers distinct advantages over traditional ground-based surveys to study the terrestrial and arboreal mammals, working effectively at night and early mornings and combined with height and certain speeds. Despite its challenges, at small to medium geographic scales, UAV thermal surveys are an up-and-coming method in the future for monitoring forest wildlife even in forests with high vegetation density. UAV as an innovative tool is very suitable for monitoring forest animals at a broader geographic scale, utilizing open areas around the forest. However, more studies are needed to test the potential use, application, and impact of this tool across species and landscapes in tropical rainforests.

\section{Acknowledgements}

We are thankful to Prof. Lilik Budi Prasetyo from the Geospatial Modeling Laboratory, Faculty of Forestry and Environment IPB, for the equipment's kind assistance during the data collection. The Ministry of Education and Culture funded this study, Republic of Indonesia (Competitive National Primary Research of Higher Education (PDUPT), with contract number 1/AMD/E1.KP.PTNBH/2020).

\section{References}

1. W. Dajun, L. Sheng, W.J. McShea, L.M. Fu, Use of remote-trip cameras for wildlife surveys and evaluating the effectiveness of conservation activities at a Nature Reserve in Sichuan Province, China, Environmental Management 38, 6 (2006) https://doi.org/10.1007/s00267-005-0302-3

2. D. A. Rahman, G. Gonzalez, S. Aulagnier, Benefit of camera trapping for surveying the critically endangered Bawean deer Axis kuhlii (Temminck, 1836), Tropical Zoology 29, 4 (2016) https://doi.org/10.1080/03946975.2016.1199763

3. J. Linchant, J. Lisein, J.S. Ngabinzeke, P. Lejeune, C. Vermeulen, Are unmanned aircraft systems (UASs) the future of wildlife monitoring? A review of accomplishments and challenges, Mammal Review 45, 4 (2016) https://doi.org/10.1111/mam.12046

4. K.S. Christie, S.L. Gilbert, C.L. Brown, M. Hatfield, L. Hanson, Unmanned aircraft systems in wildlife research: Current and future applications of a transformative technology, Frontiers in Ecology and The Environment 14, 5 (2016) https://doi.org/10.1002/fee. 1281

5. A. Barnas, D. Chabot, A.J. Hodgson, D.W. Johnston, D.M. Bird, S.N. Ellis-Felege, A standardized protocol for reporting methods when using drones for wildlife research, Journal of Unmanned Vehicle Systems 8, 2 (2020) https://doi.org/10.1139/juvs-2019$\underline{0011}$

6. C. Vermeulen, P. Lejeune, J. Lisein, P. Sawadogo, P. Bouché, Unmanned Aerial Survey of Elephants, PLoS ONE. 8, 2 (2013) https://doi.org/10.1371/journal.pone.0054700

7. W.Y. Lee, M Park, C-U Hyun, Detection of two Arctic birds in Greenland and an endangered bird in Korea using RGB and thermal cameras with an unmanned aerial vehicle (UAV), PLoS ONE. 14, 9 (2019) https://doi.org/10.1371/journal.pone.0222088 
8. J. Linchant, S. Lhoest, S. Quevauvillers, P. Lejeune, C. Vermeulen, J.S. Ngabinzeke, B.L. Belanganayi, W. Delving, P. Bouche, UAS imagery reveals new survey opportunities for counting hippos, PLoS ONE. 13, 11 (2018) https://doi.org/10.1371/journal.pone.0206413

9. M. Ancrenaz, O. Gimenez, L. Ambu, K. Ancrenaz, P. Andau, B. Goossens, J. Payne, A. Sawang, A. Tuuga, I. Lackman-Ancrenaz, Aerial surveys give new estimates for orangutans in Sabah, Malaysia, PLoS Biology 3, 1 (2005)

https://doi.org/10.1371/journal.pbio.0030003

10. A.C. van Andel, S.A Wich, C. Boesch, L.P. Koh, M.M. Robbins, J. Kelly, H.S. Kuehl, Locating chimpanzee nests and identifying fruiting trees with an unmanned aerial vehicle, American Journal of Primatology 77, 10 (2015) https://doi.org/10.1002/ajp.22446

11. R. Kays, J. Sheppard, K. Mclean, C. Welch, C. Paunescu, V. Wang, G. Kravit, M. Crofoot, Hot monkey, cold reality: surveying rainforest canopy mammals using dronemounted thermal infrared sensors, International Journal of Remote Sensing 40, 2 (2018) https://doi.org/10.1080/01431161.2018.1523580

12. D. Spaan, C. Burke, O. McAree, F. Aureli, C.E. Rangel-Rivera, A. Hutschenreiter, S.N. Longmore, P.R. McWhirter, S.A. Wich, Thermal infrared imaging from drones offers a major advance for spider monkey surveys, Drones. 3, 34 (2019) https://doi.org/10.3390/drones3020034

13. G.W. Croon, D.R. Mccullough, C.E. Olson Jr, L.E. Queal, Infrared scanning techniques for big game censusing, Journal of Wildlife Management 32, 4 (1968) https://doi.org/10.2307/3799549

14. M. Lethbridge, M. Stead, C. Wells, Estimating kangaroo density by aerial survey: a comparison of thermal cameras with human observers, Wildlife Research 46, 8 (2019) https://doi.org/10.1071/WR18122

15. M. Mulero-Pázmány, R. Stolper, L.D. van Essen, J.J. Negro, T. Sassen, Remotely piloted aircraft systems as a rhinoceros anti-poaching tool in Africa, PLoS ONE. 9, 1 (2014) https://doi.org/10.1371/journal.pone.0083873

16. C. Burke, M.F. Rashman, S.N. Longmore, O. McAree, P. Glover-Kapfer, M. Ancrenaz, S.A. Wich, Successful observation of orangutans in the wild with thermalequipped drones, Journal Unmanned Vehicle Systems 7, 4 (2019) https://doi.org/10.1139/juvs-2018-0035

17. M.A. Ditmer, J.B. Vincent, L.K. Werden, J.C. Tanner, T.G. Laske, P.A. Iaizzo, D.L. Garshelis, J.R. Fieberg, Bears show a physiological but limited behavioral response to Unmanned Aerial Vehicles, Current Biology 25, 27 (2015) https://doi.org/10.1016/j.cub.2015.07.024

18. M.E. Goebel, W.L. Perryman, J.T. Hinke, D.J. Krause, N.A. Hann, S. Gardner, D.J. LeRoi, A small unmanned aerial system for estimating abundance and size of Antarctic predators, Polar Biology 38, 5 (2015) https://doi.org/10.1007/s00300-014$1625-4$

19. B.P. Semel, S.M. Karpanty, F.F. Vololonirina, A.N. Rakotonanahary, Eyes in the sky: Assessing the feasibility of low-cost, ready-to-use Unmanned Aerial Vehicles to monitor primate populations directly, Folia Primatologica 91, 1 (2019) https://doi.org/10.1159/000496971

20. E. Bennitt, H.L.A. Bartlam-Brooks, T.Y. Hubel, A.M. Wilson, Terrestrial mammalian wildlife responses to Unmanned Aerial Systems approaches, Scientific Reports 9, 2142 (2019) https://doi.org/10.1038/s41598-019-38610-x

21. A. Marino, R. Baldi, Vigilance patterns of territorial guanacos (Lama guanicoe): the role of reproductive interests and predation risk, Ethology. 114, 4 (2008) https://doi.org/10.1111/j.1439-0310.2008.01485_1.x 
22. P. Martin, P. Bateson, "Measuring behaviour, An introductory guide, 3rd edn, Cambridge, UK: Cambridge University Press (2007)

23. J.E. Malo, P. Acebes, J. Traba J, Measuring ungulate tolerance to human with flight distance: a reliable visitor management tool?, Biodiversity and Conservation 20, 14 (2011) https://doi.org/10.1007/s10531-011-0136-7

24. N.M. Schroeder, A. Panebianco, R.G. Musso, P. Carmanchahi, An experimental approach to evaluate the potential of drones in terrestrial mammal research: a gregarious ungulate as a study model, Royal Society Open Science 7, 1 (2020) https://doi.org/10.1098/rsos.191482 